\title{
Sgr A*: Observations, Models, and Imaging of the Event Horizon with VLBI
}

\author{
Heino Falcke ${ }^{1}$, Sera Markoff ${ }^{1}$, Peter L. Biermann ${ }^{1}$, Thomas P. \\ Krichbaum $^{1}$, Fulvio Melia ${ }^{2}$, Eric Agol $^{3}$, Geoffrey Bower ${ }^{4}$
}

\begin{abstract}
We show and discuss results and prospects of high-resolution imaging of the supermassive black hole candidate Sgr A*. We also briefly review the latest observational and theoretical progress for this source. The latest millimeter-VLBI observations show compact radio emission from within a region of about 15 Schwarzschild radii. This compact component is most likely responsible for the so-called sub-mm bump in the spectrum and perhaps even for the recently discovered circular polarization discovered up to $43 \mathrm{GHz}$ and some X-ray emission through synchrotron self-Compton emission. Most importantly, however, the sub-mm emission from Sgr A* opens the door to observe, for the first time, the event horizon of a black hole directly with VLBI at sub-mm wavelengths.
\end{abstract}

\section{Introduction}

The mass of Sgr $A^{*}$ in the Galactic Center is by now relatively securely determined to be $\sim 3 \cdot 10^{6} M_{\odot}$ (Eckart \& Genzel 1997; Ghez et al. 1998). While all evidence we have so far points to the presence of a supermassive black hole, a final proof is not yet available. Similarly, the nature of processes producing the Sgr $A^{*}$, radio and X-ray, emission is still under deliberation (see Melia \& Falcke 2001 for a review). One important question is whether the radiation from Sgr $\mathrm{A}^{*}$ we see is actually produced in plasma being accreted (Melia 1992; Narayan et al. 1998) or in plasma ejected via a jet (Falcke, Mannheim, \& Biermann 1993): In either case Sgr $A^{*}$ will serve as an important test bed for the large number of low-luminosity accreting black holes in general.

To learn more about the source, we need to intensify theoretical and observational efforts. Indeed, substantial progress has again been made in the recent past and, as we discuss later on, there is even hope to eventually 'see' the event horizon of the black hole with high resolution imaging in the not-too-distant future and to uniquely demonstrate its existence.

\footnotetext{
${ }^{1}$ Max-Planck-Institut für Radioastronomie, Auf dem Hügel 69, D-53121, Bonn, Germany

${ }^{2}$ Physics Department and Steward Observatory, The University of Arizona, Tucson, AZ 85721

${ }^{3}$ Theoretical Astrophysics, Caltech MS 130-33, 1200 E. California Blvd., Pasadena, CA 91125

${ }^{4}$ Radio Astronomy Laboratory, University of California, Berkeley, CA 94720
} 

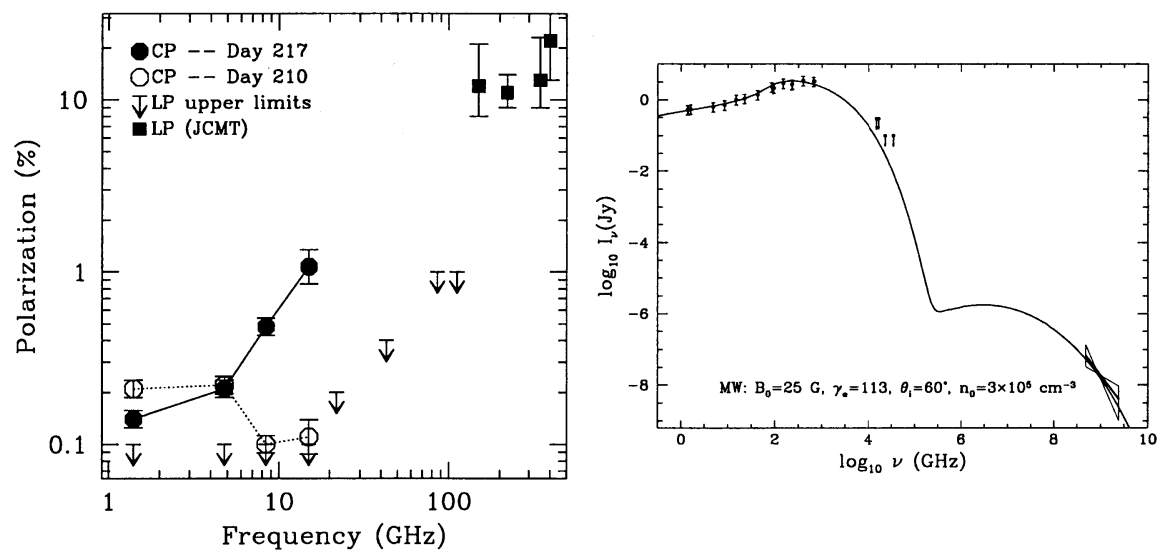

Figure 1. Left: Fractional linear (LP) and circular (CP) polarization in Sgr $\mathrm{A}^{*}$ from 1.4 to $86 \mathrm{GHz}$ from the VLA (Bower et al., in prep.; see also Bower 2000) and from JCMT at higher frequencies as claimed by Aitken et al. (2000). The sign of CP has been flipped. Right: Broad-band spectrum of Sgr A* produced by a jet model from synchrotron and synchrotron self-Compton emission. The size of the jet nozzle is $\sim 4$ Schwarzschild radii (cnf. Falcke \& Markoff 2000).

\section{Observational and Theoretical Progress}

VLBI observations have continued to narrow the size and structure of Sgr $\mathrm{A}^{*}$. Radio proper motion studies of Sgr A* (Backer \& Sramek 1999; Reid et al. 1999) indicate that the source is indeed at the Galactic Center and that it must have at least several thousand solar masses. The source has been detected with VLBI up to a frequency of $215 \mathrm{GHz}$ (Krichbaum et al. 1998) showing that the radio emission in the mm-wave regime stems from a region just tens of Schwarzschild radii across (Krichbaum et al. 1998). Observations at $43 \mathrm{GHz}$ have found tentative evidence for an intrinsic north-south elongation and new $86 \mathrm{GHz}$ observations (Doeleman et al. 2000) give an intrinsic source size of $<0.25 \times 0.13$ mas. Consistent with this, the rising spectrum at mm-to-sub$\mathrm{mm}$ wavelengths indicates that the emission at these wavelengths is extremely compact and becomes optically thin somewhere in the sub-mm regime (Melia 1992; Falcke et al. 1998).

Besides the new structural information, the discovery of circular polarization in Sgr A* (Bower, Falcke, \& Backer 1999) has revived interest in the polarization properties of Sgr $A^{*}$. It seems that $\operatorname{Sgr} A^{*}$ shows no linear polarization up to $112 \mathrm{GHz}$ at limits ranging from $0.1-1 \%$, while circular polarization has now been detected up to $43 \mathrm{GHz}$ (see Fig. 1). Aitken et al. (2000) claim a detection of linear polarization in the mm-wave regime which they interpret as confirmation for a very compact, self-absorbed emission region.

The level of circular polarization in Sgr $A^{*}$ is not unusual compared to those found in compact radio cores of jets in quasars (Wardle \& Homan 2000). 
However, the fact that circular polarization exceeds linear by a large factor, is unusual. The interpretation is unclear but certainly implies a large number of low-energy electrons either in the source (see for example the discussion in Bower, Falcke, \& Backer 1999) or along the line of sight (Blandford, this conference). In any case, Wardle \& Homan (2000) find that the circular polarization of AGN jets is always associated with the most compact component, i.e. the core.

In addition to these new radio astronomical results, recently Baganoff et al. (2000) detected Sgr A* with the X-ray satellite Chandra. The emission is rather dim which reduces significantly the accretion rates in various accretion models. The rather soft (steep) spectrum can be explained naturally in terms of synchrotron self-Compton (SSC) emission from the sub-mm bump emission region (Beckert \& Duschl 1997; Falcke \& Markoff 2000; Melia, Liu, \& Coker 2000). Within the jet model for Sgr A* the sub-mm bump is attributed to radio emission from the jet nozzle. SSC emission is then almost unavoidable and follows naturally, given the small source size at these high frequencies (Falcke \& Markoff 2000). A broadband spectrum produced by such a jet+nozzle model is shown in Fig. 1. The 'nozzle' has several physical features in common with the inner region of an accretion flow, so it is not yet possible to distinguish between these two geometries on the basis of the sub-mm/X-ray emission alone (or to say whether there is a difference at all). It may eventually be possible to do so when this is combined with the constraints due to the polarization measurements.

\section{Imaging the Event Horizon}

As outlined above, various lines of arguments directly or indirectly point to the $\mathrm{mm}$ and sub-mm emission of Sgr $\mathrm{A}^{*}$ coming from a very compact region. VLBI observations, modeling of radio and X-ray emission, and tentatively also the polarization properties all point to Sgr A* being only a few Schwarzschild radii in diameter. This is certainly a regime where strong gravity effects are important. Falcke, Melia, \& Agol (2000) have shown that if Sgr A* is surrounded by a transparent radiating plasma, the presence of an event horizon will cast a shadow on the emission region, roughly 5 Schwarzschild radii in diameter. For a $\sim 3 \cdot 10^{6} M_{\odot}$ black hole at a distance of $8 \mathrm{kpc}$ this corresponds to $37 \mu$-arcseconds. Current VLBI-experiments at $3 \mathrm{~mm}$ have already reached a resolution of $50 \mu$ arcseconds (Rantakyro et al. 1998). Given the expected resolution of $\mathrm{mm}$ - and sub-mm VLBI-experiments and the extrapolated interstellar scatter broadening one can show that the shadow of the event horizon will be detectable with VLBI at wavelengths shortwards of $1.3 \mathrm{~mm}$. Figure 2 shows an example for a rotating black hole with a very concentrated emission region.

Alternative suggestions to image this shadow with X-ray satellites have been made at this conference' (Cash et al. 2000). If the (faint) X-ray emission in Sgr $\mathrm{A}^{*}$ is indeed due to $\mathrm{SSC}$ it will be spatially coincident with the radio plasma and hence allow us to see this effect perhaps even with the resolution of the first generation of X-ray interferometers provided they will have the necessary sensitivity. On the other hand, radio astronomers have a large head start given the evolution of VLBI in the past decades. In any case, detecting the 'shadow' with the size predicted by General Relativity will provide us with the final and an unambiguous proof for the existence of an event horizon in Sgr A*. 

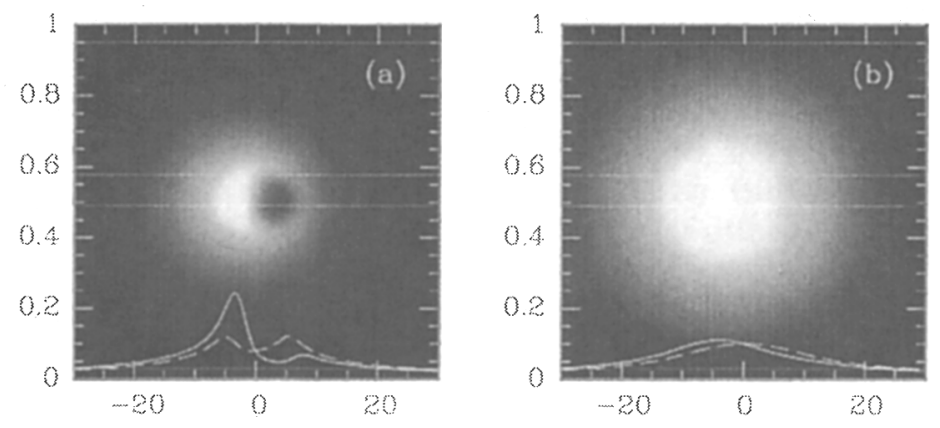

Figure 2. The shadow of the event horizon calculated for Sgr A* in the Galactic Center smoothed to the resolution of a VLBI array at 0.6 (left) and $1.3 \mathrm{~mm}$ (right). Lines give vertical (dashed) and horizontal (solid) cuts through the intensity distribution. The vertical axis is in units of $G M / c^{2}$. The simulations were made as in Falcke, Melia, Agol (2000), for a maximally rotating black hole with rotating ("Keplerian") shells and an $r^{-2}$ emissivity seen at a $45^{\circ}$ inclination angle.

\section{References}

Aitken, D. K., Greaves, J., Chrysostomou, A., et al. 2000, ApJ, 534, L173

Backer, D. C., \& Sramek, R. A. 1999, ApJ, 524, 805

Baganoff, F., et al. , L. 2000, ApJ, submitted

Beckert, T., \& Duschl, W. J. 1997, A\&A, 328, 95

Bower, G. C. 2000, GCNEWS, 11, 4

Bower, G. C., Falcke, H., \& Backer, D. C. 1999, ApJ, 523, L29

Cash, W., Shipley, A., Osterman, S., \& Jay, M. 2000, Nature, 407, 160

Doeleman, S., Shen, Z.-Q., Rogers, A. E. E., et al. 2000, ApJ, submitted

Eckart, A., \& Genzel, R. 1997, MNRAS, 284, 576

Falcke, H., Goss, W. M., Matsuo, H., et al. 1998, ApJ, 499, 731

Falcke, H., Mannheim, K., \& Biermann, P. L. 1993, A\&A, 278, L1

Falcke, H., \& Markoff, S. 2000, A\&A, 362, 113

Falcke, H., Melia, F., \& Agol, E. 2000, ApJ, 528, L13

Ghez, A. M., Klein, B. L., Morris, M., \& Becklin, E. E. 1998, ApJ, 509, 678

Krichbaum, T. P., Graham, D. A., Witzel, A., et al. 1998, A\&A, 335, L106

Melia, F. 1992, ApJ, 387, L25

Melia, F., \& Falcke, H. 2001, ARA\&A, 39, submitted

Melia, F., Liu, S., \& Coker, R. 2000, ApJLetters, in press

Narayan, R., Mahadevan, R., Grindlay, J. E., et al. 1998, ApJ, 492, 554

Rantakyro, F. T., et al. 1998, A\&AS, 131, 451

Reid, M. J., Readhead, A. C. S., Vermeulen, R. C., et al. 1999, ApJ, 524, 816

Wardle, J. F. C., \& Homan, D. C. 2000, in "Particles and Fields in Radio Galaxies", ASP Conference Series, in press 